\title{
Determination of lodoamino Acids and Inorganic lodide by Thin-Layer Chromatography
}

\author{
CHUNG WU AND RONALD C. LING \\ Department of Internal Medicine, The University of Michigan Medical School, \\ Ann Arbor, Michigan 48104
}

Received February 12, 1970

Many methods for the determination of iodinated compounds in thyroid extracts by paper and thin-layer chromatography have been reported. Some of them do not separate inorganic iodide (1-3), diiodothyronine $(4-8)$, or mono- and di-iodotyrosines $(6,9)$. Others use more than one solvent system $(4,7,10-12)$, usually one system for iodotyrosines and another for iodothyronines. Still others show poor separation of the compounds $(2,7)$, or are not supported by adequate recovery data $(1-3,5-9,11,12)$. However, good separation $(3,5,10,11)$ and recovery $(4,10)$ have been achieved in several laboratories. In our study of the synthesis of iodinated compounds in thyroid tumors, we have developed a solvent system for the separation and quantitative determination of thyroidal iodine-containing compounds including inorganic iodide by thin-layer chromatography. This paper describes our method and its application to ${ }^{131} \mathrm{I}$ experiments in vivo.

\section{MATERIAIS AND METHODS}

\section{Reagents}

3-Monoiodo-L-tyrosine (MITyr) (Mann).

3,5-Diiodo-L-tyrosine (DITyr) , 3,5-diiodo-L-thyronine (DIThy) , 3,3',5triiodo-L-thyronine (TIThy), and L-thyroxine (Sigma).

tert-Butyl alcohol (Eastman).

Methyl ethyl ketone, concentrated ammonium hydroxide, $28.9 \%$, and potassium iodide (Baker).

tert-Amyl alcohol (Baker or Fisher).

Palladium chloride.

Ninhydrin (Nutritional Biochemicals).

Eastman chromatogram sheets \#6060 (Silica gel with fluorescent indicator). 


\section{Procedure}

A. Thin-layer chromatography . Eastman chromatogram sheets \#6060 were used to make ascending runs. Iodoamino acids and KI were spotted at the origin, $2 \mathrm{~cm}$ from the lower edge of the sheet. The time of spotting was kept to a minimum to avoid degrading the amino acids. The

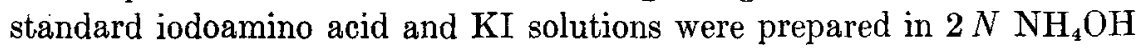
to contain 1-2 mg/ml. In spotting we used 0.5-1.5 $\mu \mathrm{g}$ MITyr, DITyr, and KI, and 1.5-2.5 $\mu \mathrm{g}$ DIThy, TIThy, and thyroxine.

The $20 \times 20 \mathrm{~cm}$ chromatogram sheets were usually cut into $10 \times 20$ $\mathrm{cm}$ strips to get an even solvent front. They were held at the top by two stainless-steel clips which were in turn strung through two parallel stainless-steel wires $4 \mathrm{~cm}$ apart. Glass tubing $1 \mathrm{~cm}$ in length was inserted as a spacer between two successive clips. With stainless-steel supporting frame $20 \times 16 \times 22 \mathrm{~cm}$ high, a total of four parrallel wires and 20 clips were mounted. A maximum of 10 sheets of $10 \times 20 \mathrm{~cm}$ each can be hung on the frame.

A solvent system consisting of $295 \mathrm{ml}$ tert-butyl alcohol, $160 \mathrm{ml}$ tertamyl alcohol, $75 \mathrm{ml} 28.9 \% \mathrm{NH}_{4} \mathrm{OH}, 35 \mathrm{ml}$ methyl ethyl ketone, and $85 \mathrm{ml}$ distilled water was prepared and mixed thoroughly. It was allowed to stand for a few minutes, and the entire content was then poured into a Pyrex rectangular jar $24 \times 18 \times 32 \mathrm{~cm}$ high with a flat ground edge. The supporting frame with chromatogram sheets was lowered into the jar. The height of the sheets should be so adjusted beforehand that about $0.7 \mathrm{~cm}$ was submerged in the solvent. A piece of filter paper moistened with about $2 \mathrm{ml} 28.9 \% \mathrm{NH}_{4} \mathrm{OH}$ was laid flat on the top of the rack. The jar was covered and sealed with a piece of ground-glass plate. It was kept at $26^{\circ}$.

The time it took for a complete run varied somewhat with the chromatogram sheets. It took less time and gave better separations with the sheets purchased in 1968 than those bought in 1969. With the "newer" sheets, however, it took $10 \mathrm{hr}$ for the solvent front to travel $15 \mathrm{~cm}$, at which point the run was discontinued. After drying the sheets in air, we sprayed them with $0.3 \%$ ninhydrin in $n$-butyl alcohol/glacial acetic acid $(100: 3)$ or viewed them under the ultraviolet light to mark the spots of iodotyrosines and iodothyronines. The iodide spot was sprayed with $0.1 \% \mathrm{PdCl}_{2}$ to give a brown color. The spots were cut and eluted in $4 \mathrm{ml}$ water. The eluates were centrifuged, if necessary. The compounds were determined quantitatively in recovery experiments at their respective absorption maxima as follows: MITyr, $305 \mathrm{~m} \mu$; DITyr, 223 and 310 (minor peak) m ; DIThy, $224 \mathrm{~m} \mu$; TIThy, 224 and 320 (minor peak) $\mathrm{m} \mu$; thyroxine, 226 and 325 (minor peak) $\mathrm{m}_{\mu}$; and $\mathrm{KI}, 226 \mathrm{~m}_{\mu}$. 
B. Labeling experiments with ${ }^{131} \mathrm{I}$. About $300 \mu \mathrm{Ci}{ }^{131} \mathrm{I}$ was injected subcutaneously into a rat. At the end of $24 \mathrm{hr}$, the rat was killed by means of an overdose of pentobarbital. The thyroid glands and a piece of neck muscle serving as a control were dissected. A homogenate of thyroid tissue in ice-cold distilled water was prepared to contain 20 $\mathrm{mg} / \mathrm{ml}$. A sufficient quantity of $15 \%$ trichloroacetic acid was added so that the final concentration was $7 \%$. After centrifugation at $27,000 \mathrm{~g}$ for $15 \mathrm{~min}$, the supernatant fluid was collected. The precipitate was washed with $0.5 \mathrm{ml}$ cold distilled water and centrifuged again. This process of washing was repeated once. The two washings and the original fluid were combined and brought to dryness under reduced pressures. The residue was dissolved in sufficient $10 \%$ isopropyl alcohol in $2 \mathrm{~N}$ $\mathrm{NII}_{4} \mathrm{OH}$ to give a concentration equivalent to $30 \mathrm{mg}$ thyroid tissue per milliliter.

Aliquots of the tissue extract, usually $1-3 \mu \mathrm{l}$, were applied on the chromatogram sheet. Unlabeled iodoamino acids and KI were added to the origin as carriers. The chromatograms were developed as described in the preceding section. The radioactive spots corresponding to the carriers and the area between two such spots were cut and placed in disposable test tubes (Packard Instruments Co.) for radioactivity counting. Direct gamma counting was done with a Packard autogamma counter.

\section{RESULTS}

Figure 1 shows the separation of MITyr, DITyr, DIThy, TIThy, thyroxine, and iodide on silica gel chromatogram. Their $R_{f}$ values with the "newer" sheets are as follows: 0.19 (DITyr), 0.23 (MITyr), 0.48 (thyroxine), 0.53 (iodide), 0.59 (TIThy), and 0.66 (DIThy). When tyrosine and thyronine are present, tyrosine will appear between MITyr and thyroxine, and thyronine, between iodide and TIThy on the chromatogram. The "older" chromatogram sheets yielded better separation of the compounds in one-half as much time, presumably because the particles used to prepare the sheets were not so fine.

To test the suitability of the method for quantitative determinations, we determined the recovery of each compound after thin-layer chromatography. The recovery values were similar whether the compounds were applied individually or in combination, and whether the compounds were added to a thyroid gland extract or not. Table 1 shows the results. For the two iodotyrosines, thyroxine, and inorganic iodide, the recoveries were excellent. Slightly lower recoveries, however, were obtained for the two iodothyronines.

We have used this method extensively to study incorporation of ${ }^{131} \mathrm{I}$ 


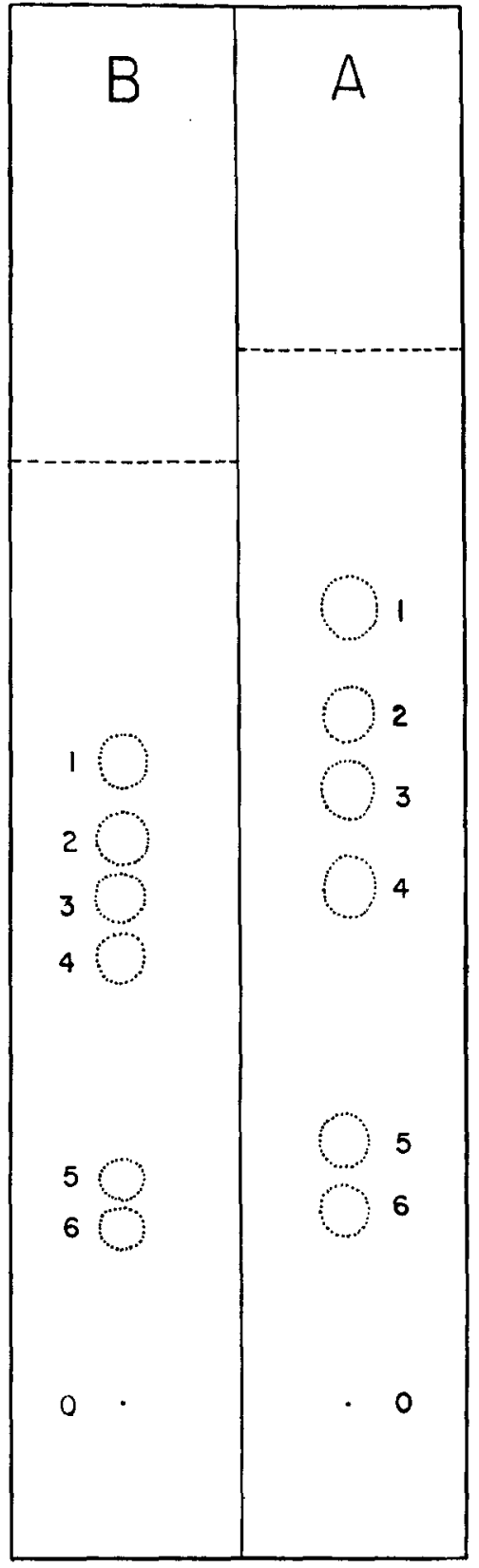

FIG. 1. Diagram of chromatograms of iodoamino acids and inorganic iodide on (A) "older" sheet, $5 \mathrm{hr}$, and (B) "newer" sheet, $10 \mathrm{hr}$. The numbers identify: DIThy (1), TIThy (2), iodide (3), thyroxine (4), MITyr (5), and DITyr (6). 


\section{TABLE 1}

\section{Recovery of Iodoamino Acids and Related Compounds after Chromatography}

After chromatography, an amount of the compound equal to that previously applied at the origin was applied to an area adjacent to the spot of that compound on the chromatogram. Both spots were then cut and the compound extracted in $4 \mathrm{ml}$ water. The amount in each extract from the chromatographed spot and from the newly applied spot was determined from a standard curve, which is linear at least through $30 \mu \mathrm{g} / \mathrm{ml}$ for each of these compounds. From these values percent recovery was calculated. Earlier, we had determined that the extraction was quantitative.

\begin{tabular}{lcccc}
\hline Compound & $\begin{array}{c}\text { Wavelength } \\
\text { used, m } \mu\end{array}$ & $\begin{array}{c}\text { Amt. before } \\
\text { chromatog., } \mu \mathrm{g}\end{array}$ & $\begin{array}{c}\text { Amt. after } \\
\text { chromatog., } \mu \mathrm{g}\end{array}$ & \% recovery \\
\hline MITyr & 305 & 60.0 & 58.8 & 98.0 \\
DITyr & 223 & 60.0 & 59.0 & 98.3 \\
DIThy & 224 & 10.0 & 9.4 & 94.0 \\
TIThy & 224 & 10.0 & 9.2 & 92.0 \\
Thyroxine & 226 & 10.0 & 10.0 & 100.0 \\
KI & 226 & 12.0 & 11.9 & 99.2 \\
Tyrosine & 281 & 20.0 & 18.8 & 94.0 \\
Thyronine & 230 & 20.0 & 18.6 & 93.0 \\
\hline
\end{tabular}

into iodotyrosines and iodothyronines by thyroid gland and thyroid tumors of the rat under different conditions. Table 2 illustrates the information that can be obtained from this kind of experiment. The experiment was carried out as described under "Procedure." After each compound has been counted, the data in counts per minute per milligram tissue were converted to percent of total counts in the thyroid extract, of which an aliquot was also used in counting. Table 2 lists the values obtained from 6 tumor-bearing rats together with the means and standard

TABLE 2

Incorporation of ${ }^{131}$ into Iodoamino Acids by Thyroid Gland of Rat Bearing a Transplantable Thyroid Tumor

The values given are expressed as percent of total radioactivity present in the thyroid extract.

\begin{tabular}{|c|c|c|c|c|c|c|c|c|}
\hline \multirow[b]{2}{*}{ Compounds } & \multicolumn{6}{|c|}{ Rat } & \multirow[b]{2}{*}{ Mean } & \multirow[b]{2}{*}{ S.D. } \\
\hline & 1 & 2 & 3 & 4 & 5 & 6 & & \\
\hline DIThy & 1.0 & 1.2 & 1.4 & 1.4 & 1.2 & 1.1 & 1.2 & 0.2 \\
\hline TIThy & 13.1 & 10.8 & 8.6 & 6.2 & 10.7 & 12.1 & 10.2 & 2.5 \\
\hline Iodide & 30.5 & 26.7 & 31.4 & 33.0 & 32.5 & 27.4 & 30.2 & 2.6 \\
\hline Thyroxine & 4.7 & 4.6 & 4.6 & 4.0 & 4.0 & 4.1 & 4.3 & 0.3 \\
\hline MITyr & 5.9 & 4.7 & 4.8 & 5.5 & 5.8 & 5.4 & 5.4 & 0.5 \\
\hline DITyr & 12.4 & 16.6 & 15.7 & 14.7 & 12.2 & 12.7 & 14.0 & 1.9 \\
\hline Total & 67.6 & 64.6 & 66.5 & 64.8 & 66.4 & 62.8 & 65.4 & \\
\hline
\end{tabular}


deviations. As we would have expected, about one-third of the total radioactivity in the thyroid extract was recovered as inorganic iodide. Some 10 to $14 \%$ each of ${ }^{131} \mathrm{I}$ in the extract appeared as TIThy and DITyr. Approximately 5\% each was incorporated into MITyr and thyroxine. DIThy had the lowest incorporation of about $1 \%$.

The sum of these percentages from the 6 compounds is about $65 \%$. This leaves $35 \%$ of the total radioactivity in the extract not accounted for by the known compounds. Most of the radioactivity unaccounted for appeared at the origin and in the area between the origin and DITyr, though low levels of radioactivity were detected in areas in between two successive known spots. This portion of the total radioactivity could vary with the conditions of the experiment. Two factors may affect the outcome. First, the recovery of DIThy and TIThy was less than quantitative (Table 1). Second, the concentration of trichloroacetic acid used to prepare the extract may not precipitate certain ${ }^{131} \mathrm{I}$-labeled proteinlike substances. This seems probable from the presence of radioactivity at the origin and in the area immediately above it.

\section{DISCUSSION}

The solvent system used in the present method contains several components. The proportions of these components have been so adjusted that the system gives a satisfactory separation of all the compounds. On the other hand, if only the iodotyrosines or the iodothyronines are to be studied, the system can be modified to give an even better separation of the group of compounds under consideration. Thus, increasing the proportion of tert-amyl alcohol increases the $R_{f}$ value for iodide, shortens the running time, and is good for separation of DIThy from TIThy. Ammonium hydroxide slows down the rate of movement of the iodide spot, thus improving the separation of TIThy from iodide. Increasing the proportion of methyl ethyl ketone also shortens the running time and makes good separation of MITyr from DITyr. The ketone, however, speeds the movement of iodide and interferes with separation of TIThy from DIThy and of TIThy from iodide. Increasing the proportion of tert-butyl alcohol or of water lengthens the running time but results in good separation of the iodotyrosines. In addition, water slows down the movement of iodide and facilitates TIThy separation. In this respect, its effect is like that of $\mathrm{NH}_{4} \mathrm{OH}$.

\section{SUMMARY}

A method has been presented for the separation of monoiodotyrosine diiodotyrosine, diiodothyronine, triiodothyronine, thyroxine, and inorganic iodide from one another by thin-layer chromatography on silica 
gel. The solvent system consisted of tert-butyl alcohol, tert-amyl alcohol, $28.9 \% \mathrm{NH}_{4} \mathrm{OH}$, methyl ethyl ketone, and water (295:160:75:35:85). Recovery of the iodo compounds from chromatography gave satisfactory results. The method has been applied to study incorporation of ${ }^{131} \mathrm{I}$ into these compounds by rat thyroid gland. Possible alterations of the five components in the solvent system to achieve certain special purposes are discussed.

\section{ACKNOWLEDGMENTS}

This work was supported in part by a grant from the University of Michigan Cancer Research Institute and by a grant from the Office of Research Administrations the University of Michigan.

\section{REFERENCES}

1. Sch Neider, G., ANd Schneider, C., Z. Physiol. Chem. 332, 316 (1963).

2. Hollingsworth, D. R., Dillakd, M., and Bondy, P. K., J. Lab. Clin. Med. 62, 346 (1963).

3. Gries, G., PFeffer, K. H., ANd Z Zappi, E. J., Klin. Wochschr. 43, 515 (1965).

4. Kologlu, S., Schwartz, H. L., and Carter, A. C., Endocrinology 78, 231 (1966).

5. Mande, R. H., and Block, R. J., Arch. Biochem. Biophys. 81, 25 (1959).

6. Heider, J. G.. and Bronk, J. R., Biochim. Biophys. Acta 95, 353 (1965).

7. FrEY, H., Scand. J. Clin. Lab. Invest. 16, 470 (1964).

8. Ouellette, R. P., and Balcius, J. F., J. Chromatog. 24, 465 (1966).

9. Bellabarba, D., Peterson, R. E., and Sterling, K., J. Clin. Endocrinol. Metab. 28, 305 (1968).

10. Sofianides, T., Meloni, C. R., Alger, E., and Caxary, J. J., Proc. Soc. Exptl. Biol. Med. 123, 646 (1966).

11. Faircloth, M. A., Williams, A. D., and Florsheim, W. H., Anal. Biochem. 12,437 (1965).

12. Sleeman, H. K., and Digas, J. W., Anal. Biochem. 8, 532 (1964). 